\title{
Atuação do enfermeiro obstetra em urgências e emergências obstétricas: revisão de literatura
}

\author{
Performance of obstetric nurse in obstetrics \\ urgencies and emergencies: literature review
}

Silas Santos Carvalho ${ }^{1}$ Carolina Santos Cerqueira ${ }^{I I}$

${ }^{1}$ Universidade Estadual de Feira de Santana (UEFS), Feira de Santana/ BA - Brasil.

${ }^{\text {II } U n i v e r s i d a d e ~ d o ~ E s t a d o ~ d a ~ B a h i a ~}$ (UNEB), Salvador/BA - Brasil.
Resumo Este trabalho tem por objetivo identificar as principais características do cuidado do enfermeiro no contexto das urgências e emergências. Trata-se de um estudo de pesquisa bibliográfica, cuja busca ocorreu nos portais de periódicos da Coordenação de Aperfeiçoamento de Pessoal de Nível Superior (CAPES) e Scientific Electronic Library Online (Scielo), tendo como critérios de inclusão artigos completos, gratuitos, em português, publicados no período de 2013 a 2018, e os de exclusão: estudos de revisão, relatos de caso, monografias, teses e dissertações e com duplicidades, onde apenas sete artigos compuseram a análise. Os estudos confirmam que a qualidade do pré-natal, a frequência de educação permanente e a valorização do profissional no contexto de atuação do enfermeiro refletem proporcionalmente nos setores de urgência e emergência, mesmo diante do contínuo enfrentamento de dificuldades com o predomínio do modelo biomédico. É necessária a elaboração de políticas na área da enfermagem obstétrica que sejam mais efetivas para proporcionarem o avanço contínuo da atuação dos enfermeiros no contexto de urgências e emergências obstétricas, incluindo, principalmente, condições mínimas de estrutura, insumos e trabalhadores e educação permanente de qualidade. Palavras-chave: Enfermeiro. Obstetrícia. Urgência e Emergência.

Abstract This work objective to identify the main characteristics of nursing care in the context of urgencies and obstetric emergencies. This is a study of bibliographic research, whose search took place in the portals of the Coordination for the Improvement of Higher Education Personnel (CAPES) and Scientific Electronic Library Online (Scielo), with inclusion criteria: free articles, complete, in portuguese, published between 2013 and 2018, and those of exclusion: review studies, case reports, monographs, theses and dissertations and with duplicates, seven articles composed the analysis. Studies confirm that the quality of prenatal care, the frequency of continuing education and the appreciation of the professional in the context of the nurse's work reflect proportionally in the urgency and emergency sectors, even in the face of the continuous confrontation of difficulties with the predominance of care biomedical model. It is necessary to elaborate policies in the area of obstetric nursing that are more effective to provide the continuous progress of nurse's performance in the context of obstetrics urgencies and emergencies, including mainly, minimum structure conditions, inputs and workers and quality continuing education.

Keywords: Nurse. Obstetrics. Urgencies and Emergencies. 


\section{INTRODUÇÃO}

A gestação é um fenômeno fisiológico e na maior parte das vezes sua evolução transcorre sem intercorrências. No entanto, há uma pequena parcela de gestantes que, por apresentarem alguma doença ou agravo, desenvolvem problemas e têm maiores probabilidades de evolução desfavorável, tanto para o feto como para si. Essas constituem o grupo chamado de "gestantes de alto risco".

Embora o Brasil tenha avançado na melhoria da atenção ao parto e ao nascimento, por meio da implementação de programas de atenção à saúde da mulher, um desafio ainda permanece: reduzir a morbimortalidade materno infantil. ${ }^{2,3}$

As principais complicações, que representam quase $75 \%$ da mortalidade materna mundial,em 2018' são: hipertensão (pré-eclâmpsia e eclâmpsia), hemorragias graves e infecções (geralmente, depois do parto), complicações no parto e abortos inseguros. A maioria dessas complicações se desenvolve durante a gravidez e muitas podem ser evitadas e tratadas. Tais complicações constituem urgências e emergências obstétricas, que se caracterizam por situações de intervenção imediata por toda a equipe de saúde, uma vez que põem em risco a vida do binômio materno-fetal. ${ }^{4-7}$

O cuidado à gestante é de acentuada importância e exige uma atenção rigorosa na avaliação das condições materno-fetais. Essa avaliação é fundamental para a identificação das distorcias de parto, do sofrimento fetal, dos partos prolongados, das atonias uterinas, da detecção precoce dos sinais e sintomas da pré-eclâmpsia e eclâmpsia e da hemorragia puerperal. Sendo a equipe de enfermagem, a responsável pelo desenvolvimento dos cuidados específicos para proporcionar uma atenção integral às parturientes que desen- volvam qualquer tipo de intercorrência no ciclo gravídico-puerperal. ${ }^{6-8}$

Dentro dessa perspectiva, no contexto de urgências e emergências obstétricas, o enfermeiro como membro da equipe multiprofissional, responsável pela assistência à parturiente, tem papel fundamental no estabelecimento de um atendimento de qualidade. Salientando que seu papel não se detém apenas aos problemas físicos apresentados pelas pacientes, mas também com o olhar holístico e humano para que essa assistência seja integral e individualizada, contribuindo para que a gestante e sua família experienciem esse processo com maior facilidade e segurança. ${ }^{9}$

A enfermagem tem buscado aprimorar seus conhecimentos técnicos e científicos para a formulação de estratégias que contribuam para a melhoria da qualidade da assistência. Assim, a atuação do enfermeiro na obstetrícia se dá por meio do acompanhamento da gestante no pré-natal,no trabalho de parto, parto e pós-parto e na assistência às gestantes de alto risco na Unidade de Terapia Intensiva (UTI) materna, implicando na necessidade de um preparo clínico para identificação de problemas reais e potenciais com vistas ao manejo adequado dos diagnósticos e das diversas situações práticas, facilitando o planejamento e a implementação dos cuidados. ${ }^{9,10}$

Considerando a problemática exposta, assim como a importância do papel do enfermeiro na saúde da mulher e da criança, com suas competências e habilidades, este estudo justifica-se pela necessidade de compreender o contexto de atuação do enfermeiro diante das urgências e emergências obstétricas, a fim de qualificar o seu trabalho.

Portanto, este estudo tem o objetivo de identificar as principais características do cuidado do enfermeiro no contexto das urgências e emergências obstétricas. 


\section{Metodologia}

Trata-se de uma revisão bibliográfica, a qual é desenvolvida com base em material já elaborado e tem como vantagem possibilitar ao pesquisador uma abordagem mais ampla do assunto em relação à pesquisa direta, permitindo uma vasta visão do objeto de pesquisa e um exame do tema sob um novo enfoque, chegando a conclusões inovadoras, não sendo, dessa forma, uma repetição do que já foi dito ou escrito sobre o assunto. ${ }^{11,12}$ Embasado na Lei $n^{\circ} .9 .610$, de 19 de fevereiro de 1998, todos os direitos autorais são respeitados e devidamente citados. ${ }^{13}$

As informações para o desenvolvimento desta pesquisa foram adquiridas nos portais de periódicos da Coordenação de Aperfeiçoamento de Pessoal de Nível Superior (CA$P E S)$ e Scientific Electronic Library Online (Scielo) nos meses de julho e agosto de 2019.

Os critérios de inclusão foram artigos disponíveis gratuitamente, publicados na íntegra, no período de 2013 a 2018, em idioma português, que apresentam aproximação com o objetivo traçado nesta pesquisa. Foram excluídos os artigos que eram estudos de revisão, relatos de caso, resultados de monografias, teses e dissertações e com duplicidades entre bases bibliográficas.

Para o levantamento dos dados, foram utilizados os termos encontrados nos Descritores em Ciências da Saúde (DeCS): enfermagem, obstetrícia e assistência ambulatorial, de forma isolada e combinada. Foram encontrados 394 artigos que, após a aplicação dos critérios de exclusão, foram reduzidos a 154 publicações que visaram responder à questão norteadora do estudo: "Quais as principais características da atuação do enfermeiro no contexto das urgências e emergências obstétricas?".

A partir das referências obtidas, procedeu-se à leitura do título e resumo, com posterior seleção do material (extraindo dos estudos selecionados o problema de pesquisa). A leitura das obras selecionadas possibilitou organizar as ideais por ordem de importância e a sintetização destas. Após leitura sistemática e interpretativa, selecionou-se para a presente revisão sete artigos.

A partir dos apontamentos, foram confeccionados fichamentos estruturados que objetivaram identificar as referências consultadas, o registro do conteúdo, comentários acerca dos estudos e a ordenação dos registros. Posteriormente, os resultados foram apresentados e discutidos para a construção da síntese do conhecimento.

\section{RESUlTAdos E DISCUSSÃo}

Foram identificados 394 artigos, sendo que 154 foram selecionados para realização da triagem. Após a leitura dos títulos e resumos, 11 foram lidos na íntegra e, por fim, sete foram incluídos nesta revisão de literatura, os quais contemplaram os critérios de inclusão e exclusão preestabelecidos.

Quanto às bases de dados incluídas na pesquisa, cinco artigos foram localizados na base Scielo e dois na CAPES. Em relação ao tipo de revista em que foram publicados os estudos incluídos na revisão, verifica-se $71,4 \%$ em revista de enfermagem e $28,6 \%$ em revista interdisciplinar de saúde.

Quanto ao tipo de delineamento de pesquisa dos estudos avaliados, evidenciou-se, na amostra, $71,4 \%$ do tipo descritivo qualitativo, e $28,6 \%$ estudos analíticos quantitativos. Em relação ao período de publicação: em 2017, houve $42,9 \%$; o ano de 2016 apresentou $28,6 \%$, e nos anos de 2014 e 2018, houve 14,3\%.

No Quadro 1, para melhor visibilidade dos dados obtidos sobre a temática investigada, apresentamos as características dos artigos, como título, autores, revista e principais achados. 
Quadro 1: Síntese dos artigos analisados

\begin{tabular}{|c|c|c|c|}
\hline Título & Autores & Revista & Principais achados \\
\hline $\begin{array}{c}\text { A prática das } \\
\text { enfermeiras obstétricas } \\
\text { nas emergências } \\
\text { vinculadas ao Programa } \\
\text { Cegonha Carioca }\end{array}$ & Progianti et al. ${ }^{16}$ & $\begin{array}{l}\text { Revista de } \\
\text { Enfermagem da } \\
\text { UERJ }\end{array}$ & $\begin{array}{l}\text { Acolhimento é visto como prática } \\
\text { ambivalente; a classificação de risco e a } \\
\text { pós-consulta como práticas de apoio e } \\
\text { reforço ao atendimento médico. }\end{array}$ \\
\hline $\begin{array}{l}\text { Avaliando diagnósticos } \\
\text { e intervenções de } \\
\text { enfermagem no trabalho } \\
\text { de parto e na gestação } \\
\text { de risco }\end{array}$ & Medeiros et al. ${ }^{10}$ & $\begin{array}{l}\text { Revista Gaúcha de } \\
\text { Enfermagem }\end{array}$ & $\begin{array}{l}\text { Para as gestantes de alto risco: sono e } \\
\text { repouso prejudicados (100\%), risco de } \\
\text { infecção }(81,8) \text { e ansiedade }(77,2 \%) \text {. As } \\
\text { intervenções foram: lavar as mãos }(80,8 \%) \text {, } \\
\text { identificar e acomodar no leito }(78 \%) \text {. }\end{array}$ \\
\hline $\begin{array}{c}\text { Análise dos } \\
\text { atendimentos } \\
\text { obstétricos realizados } \\
\text { pelo Serviço de } \\
\text { Atendimento Móvel de } \\
\text { Urgência }\end{array}$ & Michilin et al. ${ }^{23}$ & $\begin{array}{l}\text { Revista Brasileira } \\
\text { de Enfermagem }\end{array}$ & $\begin{array}{c}\text { Encaminhamento ao hospital de referência } \\
\text { e critérios de risco do Ministério da Saúde } \\
\text { não foram pertinentes: } 6,7 \% \text { e } 75,6 \% \text { dos } \\
\text { chamados, respectivamente. }\end{array}$ \\
\hline $\begin{array}{l}\text { O trabalho do } \\
\text { enfermeiro no pré- } \\
\text { natal de alto risco sob a } \\
\text { ótica das necessidades } \\
\text { humanas básicas }\end{array}$ & Errico et al. ${ }^{24}$ & $\begin{array}{l}\text { Revista Brasileira } \\
\text { de Enfermagem }\end{array}$ & $\begin{array}{c}\text { Utilização da consulta de enfermagem } \\
\text { considerando seu domínio das tecnologias } \\
\text { leves para dialogar com as tecnologias dura } \\
\text { e leve-dura. }\end{array}$ \\
\hline $\begin{array}{l}\text { Acolhimento do usuário } \\
\text { e classificação de } \\
\text { risco em emergência } \\
\text { obstétrica: avaliação da } \\
\text { operacionalização em } \\
\text { maternidade-escola }\end{array}$ & Figueiroa et al. ${ }^{22}$ & Escola Anna Nery & $\begin{array}{l}\text { A demanda espontânea demonstrou que } \\
56 \% \text { das usuárias foram classificadas } \\
\text { como prioridade verde, } 60 \% \text { das usuárias } \\
\text { relataram insatisfação e } 33 \% \text { dos } \\
\text { enfermeiros receberam treinamento. }\end{array}$ \\
\hline $\begin{array}{c}\text { Assistência de } \\
\text { enfermeiros na } \\
\text { síndrome hipertensiva } \\
\text { gestacional em hospital } \\
\text { de baixo risco obstétrico }\end{array}$ & Oliveira et al. ${ }^{18}$ & Revista Cuidarte & $\begin{array}{l}\text { Fatores que interferem na qualidade da } \\
\text { assistência: falta da avaliação fetal e de um } \\
\text { pré-natal de qualidade, da humanização e a } \\
\text { deficiência de conhecimentos relacionados } \\
\text { ao manuseio de equipamentos e até da } \\
\text { própria doença. }\end{array}$ \\
\hline $\begin{array}{l}\text { Assistência de } \\
\text { enfermagem à gestante } \\
\text { de alto risco sob a visão } \\
\text { do profissional }\end{array}$ & Nascimento et al. ${ }^{17}$ & $\begin{array}{l}\text { Revista Prevenção } \\
\text { de Infecção e } \\
\text { Saúde }\end{array}$ & $\begin{array}{l}\text { Seminários e capacitações são meios de } \\
\text { aperfeiçoar conhecimentos. Conhecem } \\
\text { a Sistematização da Assistência de } \\
\text { Enfermagem (SAE), porém não a utilizam. } \\
\text { Consideram ter profissionais insuficientes, } \\
\text { materiais escassos e precária estrutura } \\
\text { física. }\end{array}$ \\
\hline
\end{tabular}


A análise dos dados selecionados sobre a atuação do enfermeiro no contexto das urgências e emergências obstétricas revelou vários aspectos, os quais estão descritos a seguir.

Entre os serviços de urgência e emergência, encontra-se o atendimento em obstetrícia, que contém diversas intercorrências desde rotinas do ciclo gravídico puerperal como sangramentos, êmese gravídica, abortamento e ameaças do mesmo até as patologias graves como as síndromes hipertensivas específicas da gestação, hemorragias no pós-parto e infecção puerperal. ${ }^{14,15}$

Nesse contexto, o olhar do enfermeiro centra nos problemas da gestante de maneira ampliada e em seus sentimentos de bem-estar. Nessa perspectiva, o acolhimento passa a nortear sua prática porque está presente de modo contínuo na postura do profissional, podendo ser considerado uma ação técnico-assistencial com foco nas relações, que pressupõem um modo de operar os processos de trabalho em saúde, como a escuta, a construção de vínculo, a garantia do acesso com responsabilização e a resolubilidade da assistência prestada, e por isso mesmo, pode ser considerado uma das diretrizes de maior relevância ética e estética da Política Nacional de Humanização do SUS. ${ }^{1,16,17}$

Na prática, a maneira como é operacionalizado o módulo acolhimento demonstra que a atribuição de acolher, de efetivar a dimensão subjetiva e relacional do cuidado à gestante está sob responsabilidade do enfermeiro e limitada ao pequeno espaço de uma sala no setor de emergência da maternidade. Há uma falta de reconhecimento das condições necessárias para a atuação do enfermeiro diante do setor de emergência obstétrica. ${ }^{16}$

Desde o surgimento da profissão, o enfermeiro enfrenta dificuldades com o predomínio do modelo biomédico. Assim, apesar de possuir formação e atuação que os identifica com o ideário da humanização, no que tange a proporcionar à gestante e ao seu acompanhante um atendimento centrado na integralidade das necessidades apresentadas, esses profissionais ainda têm sua prática estruturada pela lógica de mercado e produtividade, com ênfase na medicalização e na concepção de risco, fazendo que haja tendência de transformar todas as queixas em transtorno ou doença biológica passível de tratamento e dependente das tecnologias

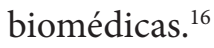

Em algumas instituições, os enfermeiros obstetras se limitam a executar ordens médicas, em outras, sua atuação é fragmentada e direcionada para o procedimento em si e não para a mulher, restringindo o profissional a ações auxiliares ao parto e a atividades mais administrativas como gerenciamento e supervisão. Ainda existem limitações quanto à execução do parto pelo enfermeiro obstetra, interferindo no processo de reconhecimento e valorização da profissão por parte da equipe médica. Os enfermeiros obstetras que atuam em maternidades-escolas sentem ainda mais essa limitação, pois, na maioria das vezes, os partos são realizados por médicos e residentes. ${ }^{8,17,18}$

No contexto da atenção básica à saúde, a qualidade da consulta de pré-natal reflete diretamente nos serviços de urgência e emergência, incluindo os de referência para obstetrícia. Nesse âmbito, ocorre a abordagem de temas essenciais para a educação em 
saúde dessas gestantes, por meio da consulta e de atividades em grupo, como: o desconforto próprio do período e maneiras de aliviá-lo, aspectos emocionais, nutrição adequada e ganho ponderal, sexualidade, aleitamento materno, desenvolvimento fetal, movimento do feto e contrações, sinais de bem-estar fetal e de risco iminente, trabalho de parto e execução do parto, a conduta a ser adotada pela cliente na hospitalização, puerpério, retorno da mulher e seu filho para casa, planejamento familiar e a continuidade do atendimento na atenção básica à saúde. Desse modo, o intuito é a prevenção de riscos e a promoção da saúde da mulher e do neonato. ${ }^{1,17,18}$

A adesão das gestantes ao pré-natal contribui com a redução de óbitos por complicações obstétricas no Brasil, porém não é o bastante, sendo necessária a implantação de intervenções que diminuam o índice de mortalidade materna. ${ }^{1}$

Importante identificar os fatores que colaboram para o aumento das intercorrências e complicações obstétricas e, por conseguinte, da morbimortalidade materno-neonatal. Isso requer reconhecimento de situação de risco e crédito no relato da gestante por parte dos médicos e enfermeiros em urgências e emergências, comprometimento, prevenção e promoção de transformações na assistência no ciclo gravídico-puerperal.,19-21

As urgências e emergências obstétricas permitem a toda a equipe envolvida identificar os casos críticos e oferecem a oportunidade de evitar o processo de morte. Desse modo, são imprescindíveis o pronto atendimento, a adequada avaliação do quadro clínico, suporte adequado para esse atendimento, bem como, a valorização da queixa da gestante, a tomada de decisões rápidas, elencar as prioridades e a alternativa de encaminhamento para hospitais de referência. ${ }^{9,14}$

De acordo com o resultado do estudo realizado em um hospital estadual de referência em obstetrícia na cidade de Recife, os enfermeiros da classificação de risco e acolhimento se depararam com os seguintes dados: a maioria das gestantes estava no terceiro trimestre de gestação; $74,5 \%$ deram entrada no serviço por demanda espontânea e 7,4\% eram provenientes da atenção primária à saúde; $1 \%$ dos casos envolveu a categoria vermelha e $56 \%$ envolveram a categoria verde. ${ }^{22}$

Conforme outra investigação, as cinco principais queixas das gestantes que motivaram os chamados ao Serviço Móvel de Urgência (SAMU) na cidade de Botucatu-SP em 2016 foram: contração uterina, perda do tampão mucoso ou conteúdo vaginal, perda de líquido amniótico, sangramento vaginal e dor no baixo ventre ou supra púbica. ${ }^{23}$

Percebe-se em ambos os estudos que essas são, de maneira geral, queixas comuns no período gestacional e as gestantes nessas situações podem e devem ser atendidas nas unidades básicas de saúde durante o seu horário de funcionamento e após avaliação, quando necessário,serem encaminhadas para o serviço de referência, diminuindo a sobrecarga deste e fortalecendo a Rede de Atenção à Saúde (RAS). ${ }^{24,25}$

Outra pesquisa evidenciou que nas emergências obstétricas nem sempre o profissional médico está disponível, o que constitui um desafio para os enfermeiros obstetras, exigindo a responsabilização pela assistência de emergência. ${ }^{16}$ 
Estudo de Errico et al. (2018) relatou que vários registros de enfermagem se apresentaram ausentes e/ou ilegíveis, o que compromete os aspectos da prestação dos cuidados realizados pelo enfermeiro e demais membros da equipe. Dados como temperatura e dosagem de glicemia capilar praticamente não são registrados. Esses problemas podem ser minimizados por meio da educação permanente, reforçando a importância do registro correto e completo dos dados obtidos nas consultas. $\mathrm{Su}$ gere-se também que sejam feitas avaliações periódicas dos instrumentos das consultas para que sejam implementadas alterações e melhorias necessárias. ${ }^{24}$

Investigações comprovaram que, devido à falta de vivência em urgências obstétricas, alguns enfermeiros não estão totalmente habilitados para assistir uma parturiente que evolui para uma intercorrência, principalmente porque atuam em um ambiente que foi preparado para realizar partos de baixo risco e, quando se deparam com uma complicação, sentem dificuldades na execução da assistência, necessitando de treinamentos periódicos e específicos. Outro fator limitante na assistência à parturiente em situação de urgência foi o suporte e a estrutura da unidade, materiais e equipamentos apropriados e funcionantes que dificultam o processo do atendimento. ${ }^{8,23,24}$

É importante identificar a alteração das necessidades humanas básicas das gestantes, pois todos os conhecimentos e técnicas acumuladas pela enfermagem dizem respeito ao atendimento dessas necessidades afetadas. Destaca-se ainda a importância de sistematizar a assistência de enfermagem para que o cuidado profissional do enfer- meiro seja efetivo junto à gestante, realizado com base científica e com autonomia profissional. ${ }^{10,23,24}$

Pesquisas evidenciaram que a maioria das enfermeiras não utiliza a Sistematização da Assistência de Enfermagem (SAE) para orientar melhor o cuidado na assistência a gestantes de alto risco devido à grande demanda de pacientes e equipe reduzida. Todavia, o enfermeiro é responsável pelo desenvolvimento de um plano de cuidados específicos para proporcionar uma atenção integral às gestantes e, por meio da SAE, poder oferecer uma visão geral da condição tanto materna quanto fetal, favorecendo a continuidade da assistência. ${ }^{17,23,24}$

Destarte, a assistência de qualidade tem impacto positivo para o binômio materno-fetal e converge para a redução dos índices de morbimortalidade de ambos, assim como as chances de complicações e intercorrências nos processos de parto e nascimento. ${ }^{10,25,26}$

\section{CONSIDERAÇões FINAIS}

Percebe-se que o enfermeiro representa uma figura fundamental na prestação do cuidado à gestante. Apesar dos achados positivos como a execução do pré-natal, o acolhimento, o olhar holístico e a educação em saúde, os estudos dessa revisão evidenciam também que o enfermeiro enfrenta questões negativas como a resistência predominante do modelo biomédico, a deficiência de educação permanente e a insuficiente estruturação de condições de trabalho e insumos.

Uma importante lacuna identificada por esta pesquisa é a escassez de estudos referentes à temática, uma vez que se acredita que 
as publicações sejam menos frequentes, pois essa área tem sido pouco explorada no âmbito da pesquisa e, com o desenvolvimento de mais estudos, os seus resultados poderão contribuir para a resolução dos problemas.

Contudo, é necessária a elaboração de políticas na área da enfermagem obstétri- ca que sejam mais efetivas para proporcionarem o avanço contínuo da atuação dos enfermeiros no contexto de urgências e emergências obstétricas. Isso inclui, principalmente, condições mínimas de estrutura, insumos e trabalhadores e educação permanente de qualidade.

\section{REFERÊNCIAS}

1. Brasil. Ministério da Saúde. Secretaria de Atenção à Saúde. Departamento de Ações Programáticas Estratégicas. Gestação de alto risco: manual técnico. Brasília, DF, 2012, 302p.

2. Carreno I., Bonilha ALL, Costa JSD. Evolução temporal e distribuição espacial da morte materna. Rev Saúde Pública, 2014;48(4):662-700.

3. Thomas TN, Gausman J., Lattof SR, Wegner MN, Kearns AD, Langer A. Improved maternal health since the ICPD: 20 years of progress. Contraception, 2014;90(6 Suppl.):S32-8.

4. Organização Pan-Americana de Saúde- OPAS. Folha informativa - Mortalidade Materna. OPAS, 2018. Disponível em:https://www.paho.org/bra/index.php?option=com_content\&view= article\&id=5741:folha-informativa-mortalidade-materna\&Itemid=820.

5. Guedes HM, Cunha NS, Dias JA, Silva GS. Implementação de estratégias para a redução da mortalidade materna no Vale do Jequitinhonha. Rev Extensão em Foco, 2018(16):1-11.

6. Rezende J., Montenegro CAB. Obstetrícia Fundamental, 13. ed. Rio de Janeiro: Koogan, 2014.

7. Ferreira MBG, Silveira CF, Silva SR, et al. Nursing care for women with pre-eclampsia and/or eclampsia: integrative review. Rev Esc Enferm USP, 2016;50(2):320-330.

8. Cavalcante TN, Almeida Junior JJ, Silva FRS, Silva MLP, Bay Junior OG. O processo de atuação do enfermeiro na assistência à parturiente em risco habitual e nas intercorrências. Convibra, 2017.

9. Ferreira CCM, Martins AS, Valadão VL, Pimenta LDN. O perfil da equipe de enfermagem no atendimento em urgências e emergências obstétricas. Revista Fafibe, 2015;8(1):332-345.

10. Medeiros AL, Santos SR, Cabral RWL, Silva JPG, Nascimento NM. Avaliando diagnóstico e intervenções de enfermagem no trabalho de parto e na gestação de risco. Rev Gaúcha Enferm, 2016;37(3):1-9.

11. Gil AC. Como elaborar projetos de pesquisa. São Paulo: Atlas, 2010, 200p.

12. Lakatos EM, Marconi MA. Fundamentos de metodologia científica, 7. ed. São Paulo: Atlas, 2010, 320 p.

13. Brasil. Lei $\mathrm{n}^{\circ}$. 9.610, de 19 de fevereiro de 1998.Altera, atualiza e consolida a legislação sobre direitos autorais e dá outras providências, Presidência da República, Casa Civil, Subchefia para Assuntos Jurídicos. Brasília, DF, p. 26. Disponível em: http://www.planalto.gov.br/ccivil_03/leis/ 19610.htm.

14. Calegari RS, Gouveia HG, Gonçalves AC. Intercorrências clínicas e obstétricas vivenciadas por mulheres no pré-natal. Cogitare Enferm, 2016; 21(2): 01-08.

15. Carvalho SS, Oliveira BR, Nascimento CSO, Gois CTS, Pinto IO. Percepção da equipe de enfermagem sobre a implantação do setor de acolhimento com classificação de risco às gestantes. Rev Bras Saúde Mater Infant, 2018; 18(2):309-315. 
16. Progianti JM, Pereira ALF, Sé CCS. A prática das enfermeiras obstétricas nas emergências vinculadas ao Programa Cegonha Carioca. Rev Enferm UERJ, 2014;22(6):743-747.

17. Nascimento TFH,Araujo FNF, Soares NSCS, Silva FM, Santos MFD, CHAVES BJP. Assistência de enfermagem à gestante de alto risco sob a visão do profissional. Rev Prev Infec Saúde, 2018;4:6.887-95.

18. Oliveira GS, Paixão GPN, Fraga CDS, Santos MKR, Santos MA. Assistência de enfermeiros na síndrome hipertensiva gestacional em hospital de baixo risco obstétrico. Rev Cuidarte, 2017;8(2):1.561-1.572.

19. Zugaib M. Zugaib Obstetrícia, 2. ed. Barueri, SP: Manole, 2012.

20. Shiroma LMB, Pires DEP. Classificação de risco em emergência: um desafio para as/os enfermeiras/os. Enferm Foco, 2011; 2(1): 14-17.

21. Araújo SM, Silva MED, Moraes RC, Alves DS. A importância do pré-natal e a assistência de enfermagem. Veredas Favip. Rev Eletr Ciênc., 2013; 3(2).

22. Figueiroa MN, Menezes MLN, Monteiro EMLM, Aquino JM, Mendes NOG, Silva PVT. Acolhimento do usuário e classificação de risco em emergência obstétrica: avaliação da operacionalização em maternidade escola. Esc Anna Nery, 2017;21(4):107, 2017.

23. Michilin NS, Jensen R., Jamas MT, Pavelqueires S., Parada CMGL. Análise dos atendimentos obstétricos realizados pelo Serviço de Atendimento Móvel de Urgência. Rev Bras Enferm., 2016;69(4):669-675.

24. Errico LSP, Bicalho PG, Oliveira TCFL, Martins EF. O trabalho do enfermeiro no pré-natal de alto risco sob a ótica das necessidades humanas básicas. Rev Bras Enferm., 2018;71(Suppl. 3):1.335-43.

25. Brasil. Ministério da Saúde Secretaria de Políticas de Saúde, Área Técnica da Saúde da Mulher. Urgências e emergências maternas: guia para diagnóstico e conduta em situações de risco de morte materna, 2. ed. Brasília, 2000.

26. Leal MC, Pereira APE, Domingues RMSM,Theme Filha MM, Dias MAB, Nakamura-Pereira M, et al. Intervenções obstétricas durante o trabalho de parto e parto em mulheres brasileiras de risco habitual. Cad Saúde Públ., 2014, 30 Sup.: S17-S47.

\section{DADOS DOS AUTORES}

\section{Silas Santos Carvalho}

Mestre em Saúde Coletiva pela Universidade Estadual de Feira de Santana (UEFS). Hospital Regional de Ruy Barbosa. Ruy Barbosa/BA - Brasil. ssc.academico@hotmail.com

\section{Carolina Santos Cerqueira}

Enfermeira graduada pela Universidade Estadual da Bahia (UNEB). Unidade Básica de Saúde da Mangabeira de Irará. Irará/BA - Brasil. carolina.s.cerqueira@hotmail.com

Submetido em: 3-10-2019

Aceito em: 15-8-2020 